Didáctica. Lengua y literatura

ISSN: 1130-0531

http://dx.doi.org/10.5209/DIDA.57133

\title{
Sobre el papel del lenguaje en el "fracaso escolar"
}

\author{
José María Gil ${ }^{1}$
}

Recibido: 16 de mayo de 2016 / Aceptado: 13 de julio de 2017

Resumen. Hay manifestaciones visibles del "fracaso escolar" en casi todas partes: muchos chicos abandonan sus estudios antes de completar el ciclo primario o secundario, o sacan bajas calificaciones en los exámenes. Los participantes principales de este proceso son niños y adolescentes de los sectores socioeconómicos menos favorecidos y las investigaciones vienen mostrando desde hace tiempo que, en gran medida, la causa del "fracaso" es de orden social Sin embargo, ¿cumple el lenguaje algún papel en este complejo proceso de exclusión? Algunas hipótesis bastante difundidas (a veces de forma explícita y a veces implícita) se han ofrecido como respuesta. En este trabajo se las pone a prueba a partir de la lingüística sistémico-funcional (LSF). Parece que el "fracaso escolar" también está condicionado por la variedad de lengua, aunque esto no implica una deficiencia de dicha variedad. Entender este complejo fenómeno es un primer e imprescindible paso para empezar a resolver el problema.

Palabras claves: fracaso escolar; lenguaje; cultura; pobreza; dialectos sociales.

\section{[en] On the role of language in "school failure"}

Abstract. There are visible manifestations of "school failure" almost everywhere: many boys drop out of school before completing their primary or secondary school year, or they score low on exams. The main participants in this process are children and adolescents from the less favored socio-economic sectors, and research has long shown that the cause of "failure" is of a social nature. However, does language play any role in this complex process of exclusion? Some quite widespread hypotheses (sometimes explicitly and sometimes implicitly) have been offered as an explanation. In this work such hypotheses are tested on the basis of systemic-functional linguistics (SFL). It seems that "school failure" is also conditioned by the variety of language, although this does not imply an impairment of such variety. Understanding this complex phenomenon is a first and essential step to begin to solve the problem.

Key words: School failure; language; culture; poverty; social dialects

\section{[fr] Sur le rôle du langage dans «l'échec scolaire»}

Résumé. Il y a des manifestations visibles de «l'échec scolaire» presque partout: Beaucoup d'enfants abandonnent avant la fin de l'école primaire ou secondaire, ou prendre des scores faibles de test. Les principaux participants de ce processus sont les enfants et les adolescents des secteurs socio-économiques défavorisés, et la recherche montre que, en grande partie, la cause de «l'échec» est du l'ordre social. Cependant, est-ce que la langue a un rôle dans ce complexe processus d'exclusion? Certaines hypothèses assez répandues (parfois explicitement, parfois implicitement) ont été offerts en guise d'ex-

\footnotetext{
Departamento de Filosofía

Universidad Nacional de Mar del Plata-CONICET, Argentina

josemaria@gilmdq.com
} 
plication. Dans cet article, ils sont analysés à partir de la linguistique systémique fonctionnelle (LSF). Il semble que le «l'échec scolaire» est aussi conditionnée par la variété de la langue, bien que cela ne signifie pas un manque de cette variété. Comprendre ce phénomène complexe est une première et essentielle étape pour commencer à résoudre le problème.

Mots-clés: Échec scolaire; langue; culture; pauvreté; dialectes sociaux.

Sumario: 1. Introducción: Algunos problemas fundamentales en torno al fracaso escolar. 2. Un enfoque socio-funcional del lenguaje. 3. Explicaciones en torno al fracaso escolar: La hipótesis del déficit. 4. El pensamiento como posibilidad de cualquier lengua o dialecto. 5. La hipótesis de la diferencia. 6. La hipótesis del estereotipo. 7. Conclusiones. 8. Bibliografía.

Cómo citar: Gil, J. M. (2017). Sobre el papel del lenguaje en el "fracaso escolar". Didáctica. Lengua y literatura, 29, 2017, 121-137.

\section{Introducción: Algunos problemas fundamentales en torno al fracaso escolar}

Hacia el comienzo de las clases no es infrecuente que, en Argentina por ejemplo, los programas de radio y las portadas de los diarios se escandalicen por la enorme cantidad de estudiantes que desaprueban las evaluaciones de diagnóstico de la escuela secundaria y los exámenes de ingreso a las universidades. Entre otros, aparece el dictamen de que los chicos "no saben hablar bien"; esa sería una razón fundamental para entender por qué a ellos "les va tan mal en la escuela".

La creencia es todavía más lapidaria cuando se refiere a los chicos de sectores socioeconómicos menos favorecidos, como los "villeros" o los (hijos de) inmigrantes de algunos países limítrofes. Para la imaginación popular, y aun para la de los medios de comunicación, los chicos de esos grupos sociales usan un lenguaje deficitario, "pobre en recursos".

El complejo proceso que desde la década de 1970 se llama "fracaso escolar" se caracteriza por los resultados deficientes en las evaluaciones y el abandono del sistema escolar en alguno de sus niveles. Este conjunto de problemas guarda una estrecha relación con diversos aspectos de la realidad educativa de hoy. La mayoría de esos aspectos no son estrictamente lingüísticos, pero están conectados con el fracaso escolar (y por ello, aunque sea indirectamente, con el lenguaje). Las investigaciones actuales de educación reconocen de forma más o menos enfática algunos supuestos fundamentales en torno a este problema. Considérense por ejemplo los cinco temas que se enuncian a continuación.

1. El nivel socioeconómico y la pertenencia cultural se correlacionan con el rendimiento escolar. Tal como señalan Córdoba Caro et al. $(2011,91)$ para el caso del colegio secundario en Badajoz, el perfil del alumno con un alto rendimiento académico global corresponde al estudiante con un nivel económico y cultural alto.

2. El fracaso escolar se asocia mayormente a los chicos de sectores sociales menos favorecidos, por ejemplo los hijos de inmigrantes. En general, las familias inmigrantes le otorgan una gran importancia a la educación de sus hijos. Sin 
embargo, el alto nivel de deserción que hay entre ellas puede interpretarse como un indicio de que la confianza de estas familias en la escuela puede terminar deteriorándose (Santos Rego, Lorenzo Moledo y Priegue Caamaño 2011, 108). En ese sentido, el rendimiento escolar de los chicos de sectores sociales menos favorecidos va por detrás del rendimiento de sus pares de sectores socioeconómicos más acomodados.

3. El nivel socioeconómico no se correlaciona ni con la creatividad ni con la inteligencia, más allá de cómo se definan (o midan) estos dos términos. Desde luego, los puntos 1 y 2 de esta lista no implican que los chicos de los sectores menos favorecidos tengan deficiencias constitutivas en la creatividad o la inteligencia. Debe tenerse en cuenta, además, que la situación se torna aún más compleja cuando se considera, como lo hacen López-Martínez y Navarro-Lozano $(2010,294)$, que los factores de la creatividad no tienen relación con el coeficiente intelectual de una persona.

4. Los estudiantes y los docentes por lo general manifiestan algún tipo de conciencia sobre los roles que desempeñan en el proceso educativo. Así, Trevitt, Breman y Claire (2012) dan por supuesto que esto es así cuando estudian la evaluación y la auto-evaluación. En ciertas ocasiones, la conciencia sobre el rol que uno mismo desempeña y el rol que cumplen los otros puede llevar a la formación de estereotipos que terminan condicionando las conductas.

5. A pesar de lo que muchas veces se proclama, es común que la escuela no haga hincapié en la dimensión afectiva. Por ejemplo, Murillo Torrecilla y Hernández Castilla $(2011,422)$ señalan que la escuela tiene un papel limitado en el desarrollo de productos socio-afectivos. Ciertos matices afectivos o interpersonales se corresponden con alguna función del lenguaje que no es la que por lo general se privilegia en la escuela.

Las investigaciones actuales en educación manifiestan una clara tendencia a privilegiar la "validez interna", es decir, a recoger, analizar e interpretar datos de casos concretos y muy acotados que siempre pueden justificarse en los términos del contexto puntual que se ha seleccionado, por ejemplo cierta cantidad de estudiantes secundarios de ciertas escuelas de Badajoz (Córdoba Caro et al., 2011). Estas investigaciones aportan una cuantiosa y significativa información sobre problemas educativos concretos. Sin embargo, carecen de una fundamentación general para los problemas que estudian: en nombre de la peculiaridad de los contextos sociales específicos se descuidan fenómenos generales que afectan en mayor o menor medida a todas las comunidades.

En algún punto, este trabajo intentará mostrar que las investigaciones en torno al fracaso escolar pueden tener "validez externa" (pueden ser útiles para contextos sociales muy amplios) cuando se correlaciona este aspecto con el lenguaje. Dicho de otra forma, se buscará contribuir a la fundamentación general del vínculo que existe entre el fracaso escolar y el lenguaje. Concretamente, se espera mostrar qué aspectos lingüísticos inciden en el fracaso escolar. Así, las ciencias del lenguaje habrán hecho su aporte a las investigaciones que buscan no sólo entender sino también resolver uno de los problemas educativos más urgentes de la actualidad. 


\section{Un enfoque socio-funcional del lenguaje}

Dentro de la lingüística sistémica-funcional (de aquí en más, LSF) se acepta la hipótesis de que el lenguaje es un producto de la cultura, lo cual guarda una relación decisiva con el problema del fracaso escolar.

Esta concepción es diferente, y tal vez complementaria, de la de quien posiblemente sea el lingüista más famoso de la historia: Noam Chomsky. Para él, el lenguaje es una facultad innata de la mente humana que no está configurada por ni para el uso $(1995,168 ; 2000,75 ; 2003,212-213 ; 2005,2-3)$. En consecuencia, siempre según Chomsky, cualquier teoría que no conciba al lenguaje como facultad de la mente se basa en hipótesis irremediablemente falsas. La postura de Chomsky según la cual el lenguaje no debe concebirse como un sistema de comunicación ha dado lugar incluso a la crítica de eminentes investigadores generativistas, por ejemplo las de Pinker y Jackendoff (Jackendoff, 1997, 2002; Pinker, 1999; Pinker y Jackendoff, 2005). Así y todo, las hipótesis chomskyanas gozan aún de una gran aceptación en buena parte del mundo, especialmente en los Estados Unidos.

Ahora bien, la idea de que el lenguaje es parte constitutiva de la biología humana no tiene que ser incompatible con la idea de que el lenguaje es también un producto de la cultura. Así lo explica el lingüista británico Michael Halliday:

Biológicamente, todos somos semejantes, por lo que toca a la capacidad para aprender la lengua; como especie, poseemos esa capacidad, lo mismo que poseemos la capacidad para mantenernos erguidos y caminar, y eso es enteramente independiente de las habituales evaluaciones de la "inteligencia" en la forma que sea. Por otra parte, ecológicamente, cada uno de nosotros es único, puesto que el modelo ambiental nunca se repite exactamente y porque la experiencia de un individuo jamás es igual a la de nadie... Nuestro medio es conformado por la cultura y las condiciones en las que aprendemos la lengua en gran medida están determinadas culturalmente (Halliday, 1978, 35).

En síntesis, la LSF no niega que el lenguaje tenga una dimensión biológica, pero entiende que (también) es imprescindible estudiarlo en términos del uso y del contexto de la cultura.

Las hipótesis generativistas sobre el uso siempre han preocupado a muchos autores a la hora de justificar la LSF. Roger Fowler, por ejemplo, señala que la lingüística moderna presenta la paradoja de que su representante más famoso (Chomsky, claro) es mundialmente conocido como militante y activista político pero no manifiesta interés profesional alguno en el análisis de pronunciamientos, noticias o libros que, según su propia tesis, subvierten el proceso democrático. Para Fowler, si se aceptan los supuestos y el razonamiento de Chomsky, se acepta entonces la conclusión de que la LSF no es lingüística, lo cual no es un caso de juego limpio y democrático: "en el mundo más liberal de la lingüística funcional", el estudio de la comunicación "es una práctica legítima que no necesita defensa especial alguna" (Fowler, 1996, 5).

La LSF es una teoría muy compleja en cuyo seno se registran debates de importancia. Así y todo, los siguientes supuestos forman parte de lo que podría llamarse "núcleo duro de la teoría", para emplear la servicial terminología de Lakatos (1974). Todas las vertientes de la LSF aceptan las hipótesis que se enuncian a continuación: 
1. El lenguaje es una semiótica social: se trata de un sistema de significados que está configurado por la cultura y que hace que se transmitan los significados de dicha cultura.

2. El sistema lingüístico es una compleja red de opciones. La elección de una u otra opción lingüística se relaciona con necesidades comunicativas de los hablantes o escritores en ciertos contextos de situación.

3. Los productos de la conducta verbal (los textos) pueden estudiarse científicamente sin necesidad de recurrir a un modelo psicológico, cognitivo o neurológico en particular.

4. Un niño aprende la lengua bajo las condiciones de una experiencia única e irrepetible, en un determinado contexto de cultura.

5. Las necesidades comunicativas determinan el aprendizaje y la estructura del sistema lingüístico.

6. Las funciones que el lenguaje desempeña (por ejemplo, hacer referencia a lo que ocurre en el mundo mental y extra-mental) no son sólo "funciones", sino que además forman parte del sistema lingüístico: por ello son "metafunciones".

7. En relación con lo enunciado en (3), los postulados de la LSF no tienen que ser incompatibles con alguna teoría que caracterice al lenguaje en términos psicológicos, cognitivos o neurológicos.

En resumen, para la LSF, “el lenguaje es como es por lo que hacemos con él”; el enfoque puede considerarse sociolingüístico en un sentido amplio del término.

Tal vez sea conveniente dar algunos ejemplos para mostrar cómo interactúan las hipótesis del conjunto (1)-(7). Desde la perspectiva sistémico-funcional, resulta plausible la hipótesis de que la cláusula de una lengua particular, como el castellano, es una estructura sintáctica que expresa varios significados al mismo tiempo. De este modo, la cláusula castellana consta de una variada trama de significados: el "principio multifuncional del lenguaje", en palabras de Robin Fawcett $(2008,216)$, establece que toda cláusula desempeña varias funciones al mismo tiempo; es decir, una lengua natural permite expresar diversos significados de forma simultánea en una única estructura. Este principio de multifuncionalidad podría aplicarse muy probablemente a todas las lenguas humanas.

Sin entrar en detalles técnicos y debates teóricos, puede concluirse que una cláusula simple como En el Congreso se aprobó la ley expresa un significado "experiencial", un significado "interpersonal" y un significado "textual":

- En efecto, la cláusula se refiere a un hecho de la experiencia de los hablantes en el que se habla de un proceso "material" como aprobar (una ley): función experiencial.

- En lo que respecta al diálogo, la cláusula es una afirmación, el hablante o el escritor proveen información a sus oyentes o lectores: función interpersonal. En el caso de la lengua española, el modo se manifiesta por la desinencia del verbo, lo cual permite distinguir, por ejemplo, el indicativo del imperativo, es decir, un ofrecimiento de información de un pedido de acción (Gil y García 2010).

- Si se tiene en cuenta la cláusula como mensaje, ésta es acerca de la circunstancia en la que se dio el proceso de "aprobar"; "en el Congreso" aparece en 
posición inicial y cuenta como el tema ya que no es lo mismo hablar de "el Congreso" que de otro ámbito: función textual.

El esquema que aparece a continuación resume las observaciones anteriores.

\begin{tabular}{|l|l|l|l|}
\hline Metafunción & En el Congreso la ley \\
\hline Experiencial & $\begin{array}{l}\text { Dónde se hizo (cir- } \\
\text { cunstancia) }\end{array}$ & $\begin{array}{l}\text { Lo que se dio (proceso, } \\
\text { en este caso, acción ma- } \\
\text { terial) }\end{array}$ & $\begin{array}{l}\text { Qué se hizo } \\
\text { (meta) }\end{array}$ \\
\hline Interpersonal & $\begin{array}{l}\text { Modo (desinencia verbal): tercera persona del singular, pretérito } \\
\text { perfecto simple: -ó. Afirmación: ofrecimiento de información }\end{array}$ \\
\hline Textual & $\begin{array}{l}\text { Núcleo de informa- } \\
\text { ción: } \\
\text { Tema. } \\
\text { Lo que se destaca es } \\
\text { dónde pasó. }\end{array}$ & Rema \\
\hline
\end{tabular}

De esta forma, vemos cómo una cláusula simple expresa al menos tres significados de forma simultánea: experiencial, interpersonal y temático. Estos tres significados presentes en la estructura de la cláusula se correlacionan con las tres grandes funciones que el lenguaje cumple:

- metafunción experiencial, organización de la experiencia;

- metafunción interpersonal, mantenimiento de interacciones verbales;

- metafunción textual, producción de mensajes.

Recordemos que las funciones no son sólo grandes usos del lenguaje, sino también "metafunciones", puesto que forman parte del sistema lingüístico y se manifiestan en las estructuras gramaticales como la cláusula.

La teoría que respalda el análisis funcional se denomina "sistémica" porque consiste en una teoría del significado como elección. La LSF supone que el lenguaje es, al igual que otros sistemas semióticos, una compleja red de opciones de significado. El sistema lingüístico constituye un gran orden paradigmático: se organiza en términos de lo que está ausente, es decir, de relaciones "o" (qué podría estar en lugar de qué). Por su parte, la estructura lingüística (por ejemplo, la de la cláusula) es sintagmática: se organiza en términos de lo que está presente, es decir, de relaciones " $\mathrm{y}$ ".

En el sistema lingüístico global, todo sub-conjunto de alternativas y la entrada para dichas alternativas constituyen un sistema de opciones en este sentido técnico. Por ejemplo, todas las cláusulas castellanas seleccionan o no seleccionan Sujeto. En el caso (bastante más probable) de que sí se seleccione un Sujeto, este puede ser explícito o tácito. Véase al respecto la Figura 1. Hay, desde luego, más opciones para las tres entradas terminales (explícito, tácito, impersonal), pero la figura y la breve explicación de las líneas anteriores acaso sirvan para ilustrar qué es una red sistémica. 


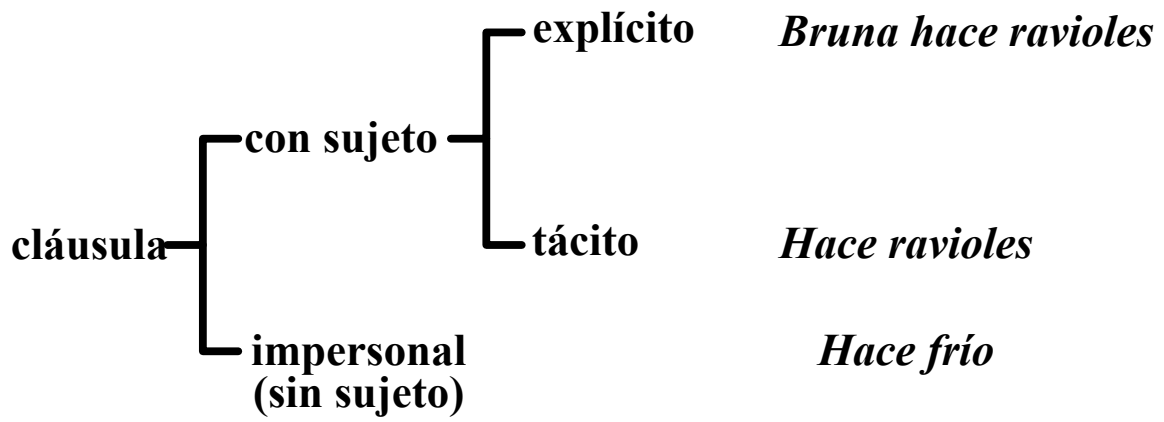

Figura 1: Sistema simplificado del sujeto gramatical en castellano.

(Con ejemplos de realización a la derecha)

Cada sistema, como el del Sujeto, es un momento de elección que contribuye a la formación de estructura. Desde luego, no se sugiere que haya aquí una opción (necesariamente) consciente. Halliday y Matthiessen $(2004,24)$ señalan que "los "momentos' son pasos analíticos en la construcción gramatical del significado". Inmediatamente después de esta definición, los autores reconocen de forma explícita que la LSF no sólo no es incompatible con una teoría lingüística de base neurológica, sino que además puede necesitarla: remiten a Lamb (1999) para ver "la relación entre la elección de significados y lo que ocurre en el cerebro". Esta cuestión es fundamental porque permite tener una visión más completa de las hipótesis de la lista de más arriba, en especial de (3) y (7). La LSF es consistente con la teoría de redes relacionales de Lamb. En este sentido, uno de los desafíos más importantes de la teoría lingüística actual es ver si las hipótesis que manejamos acerca del lenguaje pueden "reubicarse" en un modelo que busque la plausibilidad neurológica (Lamb, 2005, 2006).

En tanto teoría del lenguaje como semiótica social y como sistema de opciones, la LSF acepta la explicación de que (especialmente en lo referido a los prejuicios) la causa del fracaso escolar es social y no lingüística. Sin embargo, la LSF también reconoce que el lenguaje desempeña una función decisiva en la transmisión de valores y orientaciones comunicativas. Entonces, ¿los valores y los significados que se aprenden con el lenguaje pueden hacer que a los chicos de los sectores sociales más desfavorecidos les vaya mal en la escuela?

La teoría de Basil Bernstein (1971) ha sido definida por el mismo Halliday como "una teoría de la naturaleza y los procesos de transmisión cultural y del papel fundamental que el lenguaje desempeña en ella" (Halliday, 1978, 135). Bernstein se preocupó especialmente por el problema del "fracaso escolar", cuya manifestación más visible son los chicos a los que "les va mal" en el colegio, los que "no aprueban los exámenes" o los que, simplemente, terminan por abandonarlo antes de completar el ciclo de instrucción primaria o secundaria. A partir de trabajos como el de Bernstein, se determinó que la distribución del fracaso no es aleatoria sino que sigue pautas conocidas y "tristemente predecibles".

En el caso de los países desarrollados de Europa y de América del Norte, el fracaso escolar ha sido tradicionalmente un problema al que se enfrentan los niños de la clase trabajadora más baja en las grandes áreas urbanas. Ya a principios de la década de 1970, muchos investigadores se dieron cuenta de que no era suficiente que los ni- 
ños estuvieran bien alimentados y que pasaran una cierta cantidad de años recibiendo educación formal. El fracaso persistía.

En América Latina, en el siglo XXI, la situación no es tan distinta. No es demasiado difícil explicar por qué a un chico indigente o pobre "le va mal en la escuela". Sin embargo, el problema del fracaso escolar es todavía más amplio (en cuanto a la cantidad de individuos afectados) que "el hambre de los chicos pobres". Ahora, si la explicación del fracaso educativo no es exclusivamente la pobreza o la indigencia, ¿dónde debe buscarse una respuesta? Veamos algunas hipótesis en los incisos siguientes.

\section{Explicaciones en torno al fracaso escolar: La hipótesis del déficit}

Algunos creyeron que la respuesta estaba en el lenguaje, concretamente, en la variedad de lenguaje hablada por los grupos de chicos con mayor tendencia a "fracasar". La primera de estas versiones es la hipótesis del déficit, que establece que el dialecto (la variedad social) hablado por estos grupos tiene algún tipo de deficiencia con respecto a la lengua o el dialecto estándar, es decir, la variedad social que goza de mayor prestigio y que se emplea en ámbitos educativos, administrativos y en aquéllos que son característicos de los grupos sociales "medios" y "altos". La deficiencia del dialecto no-estándar con respecto al estándar residiría, según la hipótesis del déficit, en los sonidos, en el vocabulario, en las estructuras sintácticas o en el significado.

El planteo de la hipótesis del déficit puede sintetizarse del siguiente modo: hay ciertos grupos sociales (de clase, étnicos, familiares, regionales, etc.) cuyo dialecto es incompleto o deficiente. Si se la analiza con atención, enseguida se advierte que la hipótesis es falsa. Sin embargo, "debemos considerar seriamente esos criterios [para sostener la hipótesis del déficit]; los sostiene gente seria de buena fe, que ha pensado en el problema y está ansiosa por encontrarle solución; al mismo tiempo, debe decirse muy enérgicamente que se equivoca" (Halliday, 1978, 137).

Los especialistas en lenguaje y educación saben bien que la hipótesis del déficit es falsa. Sin embargo, en muchas instituciones educativas y en buena parte de los medios de comunicación todavía se la sostiene explícita o implícitamente. También la dan por verdadera muchas personas en sus valoraciones cotidianas sobre el lenguaje. Por ello, aunque no es necesario explicarles a los especialistas que la hipótesis del déficit es falsa, sí es importante refutarla para que muchos docentes, comunicadores y hablantes en general no la sigan aceptando y difundiendo. En efecto, muchas personas creen que los hablantes de los dialectos no estandarizados "hablan mal" y que los hablantes del dialecto estándar "hablan bien", pero esa esta discriminación se sostiene únicamente en un prejuicio de carácter sociocultural: como lamentablemente se cree que las personas de los grupos sociales menos favorecidos pertenecen a un nivel inferior, de un modo igualmente lamentable se cree que su forma de habla es inferior. La siguiente anécdota sirve de ejemplo al respecto: en un viaje de estudios a la provincia argentina de Mendoza, la directora a cargo de los chicos de un colegio de la ciudad de Buenos Aires pidió que se cambiara al guía, que era chileno, porque ella consideraba que "no sabía hablar correctamente" y que por ello podía perjudicar el aprendizaje de sus alumnos. En realidad, la única razón que tenía la directora para creer que el dialecto del guía era deficitario era el prejuicio de ella. 
En definitiva, no hay datos que permitan confirmar la hipótesis del déficit. Nada permite decir que los chicos que fracasan en la escuela tengan un vocabulario o un sistema gramatical más pobre que los que tuvieron éxito. Las razones son las siguientes:

1) No parece fácil separar el vocabulario de la gramática. En la concepción funcionalista integran un solo componente en el sistema lingüístico, a saber, el sistema léxico-gramatical.

2) Los problemas a contabilizar son tantos que difícilmente podrían evaluarse los recursos léxico-gramaticales de un individuo en términos cuantitativos.

3) Aunque se pudiera hacer la contabilidad imaginada en (2), eso diría poco del potencial lingüístico de un dialecto. De forma análoga, no se juzga a un escritor nada más que por la cantidad de palabras y estructuras sintácticas que ha usado.

En conclusión, los tres hechos mencionados permiten refutar la hipótesis. El siguiente texto presenta el argumento que se usa para esa refutación:

\section{Argumento de la refutación de la hipótesis del déficit}

- Premisa 1: Si el dialecto no-estándar $x$ es esencialmente incompleto comparado con el dialecto estándar $[H]$, entonces, si se revisa la estructura léxico-gramatical del dialecto no-estándar $x[C]$, se observará que la estructura léxico-gramatical de ese dialecto $x$ impide expresar ciertos significados que sí pueden expresarse en el dialecto estándar $[E]$.

- Premisa 2: Si se revisa la estructura léxico-gramatical del dialecto no-estándar $x[C] \mathrm{y}$, a través de ese análisis, se advierte que la estructura léxico-gramatical del dialecto no-estándar $x$ no impide expresar ciertos significados que sí pueden expresarse en el dialecto estándar [no $E]$.

- Conclusión: El dialecto no-estándar $x$ no es esencialmente incompleto comparado con el dialecto estándar [no $H]$.

La evidencia a favor de la premisa 2 es el siguiente dato: La variedad de habla no-estándar es un sistema lo suficientemente "rico" que permitiría expresar "cualquier significado". O, dicho de otro modo, quizá algo tosco: con la gramática y con las funciones del dialecto no-estándar "se puede hablar de cualquier cosa", al igual que puede hablarse de cualquier cosa con la gramática y las funciones del estándar. El vocabulario de un dialecto siempre puede ampliarse mediante préstamos o neologismos, si lo que se busca es hacer referencia a una innovación o un descubrimiento, por ejemplo. Las metafunciones lingüísticas de los diferentes dialectos, en términos globales, siempre son las mismas, y sólo se registran diferencias con respecto a las orientaciones que privilegian un dialecto u otro.

En este caso, la LSF nos permite refutar una hipótesis que, por desgracia, está muy difundida en la escuela y en los medios de comunicación.

En términos del modelo hipotético-deductivo, la forma lógica del argumento de la refutación de la hipótesis del déficit (como la de toda refutación) es la de un razonamiento válido (Hempel, 1966, 22): 
Premisa 1: $H \supset(C \supset E)$

Premisa 2: $C \cdot \sim E$

Conclusión: $\therefore \sim H$

Aquí, $H$ es la hipótesis del déficit; $C$ representa la contrastación (en este caso, la revisión de la estructura del dialecto no-estándar $x$ ) y, por último, $E$ se refiere al efecto esperable: que el dialecto no-estándar impida expresar ciertos significados. Si las dos premisas son verdaderas, la negación de la hipótesis también tiene que ser verdadera en la conclusión.

\section{El pensamiento como posibilidad de cualquier lengua o dialecto}

Esta cuestión ha sido claramente explicada, entre otros, por Emile Benveniste: el pensamiento es universal y trasciende los condicionamientos de una lengua o de un dialecto. El pensamiento adopta su propio curso más allá de las estructuras particulares de cada sistema lingüístico.

El pensamiento chino bien pudo haber engendrado categorías tan específicas como el tao, el yin y el yang: no es menos capaz de asimilar los conceptos de la dialéctica materialista o de la mecánica cuántica sin que sea obstáculo la estructura de la lengua china (Benveniste, 1958, 73).

Así, una lengua o un dialecto nunca pueden ser un obstáculo para el pensamiento o para la concepción de ciertos significados. Aunque la lengua o el dialecto puedan favorecer cierto tipo de pensamiento, no impedirán ni favorecerán la actividad del pensamiento como tal. El "vuelo del pensamiento" está ligado a la capacidad de la mente humana. Y, como sugería Sapir (1921), el cerebro de un individuo está perfectamente pre-dispuesto para aprender cualquier lengua y, por lo tanto, para entender cualquier pensamiento. De manera análoga, la capacidad del pensamiento es universal porque el sistema lingüístico es una estructura de significado; Benveniste (1958, 74) llega a decir que "pensar es manejar los signos de la lengua". No puede afirmarse que entendérselas con los signos de una variedad no prestigiosa signifique estar más lejos del pensamiento o en un nivel inferior del pensamiento.

\section{La hipótesis de la diferencia}

La LSF permite preguntarnos (y tal vez responder) si la causa del fracaso escolar efectivamente está en el lenguaje, pero no se sostiene aquí que el dialecto de estos grupos con "tendencia a fracasar" sea deficitario. Podemos, en este contexto, limitarnos a decir que la variedad de habla de los grupos desprestigiados o estigmatizados es tan sólo distinta. Esto efectivamente parece ser así: el dialecto de algunos niños es diferente del de otros.

Ahora bien, si ningún dialecto es peor que el estándar, ¿por qué el no-estándar está en desventaja? En principio, la respuesta es simple: el niño que habla el dialecto no-estándar se encuentra en desventaja porque ciertos contextos exigen el uso del 
dialecto estándar. En efecto, el maestro, la asignatura, el sistema en general, son los que lo exigen. De este modo, el chico de un grupo social desfavorecido, como un "villero" por ejemplo, se encuentra con que en la escuela se usa una variedad que él no maneja; este chico debe adaptarse a un proceso educativo o a un modo de vida que se desarrolla casi enteramente en el dialecto estándar. La carencia de este chico es meramente práctica, pero se trata de una falta que de hecho sí lo pone en desventaja.

Con respecto al término "villero" quizá es necesaria una aclaración porque, lamentablemente, tiene connotaciones peyorativas y en Argentina es casi imposible usarlo sin que genere sonrisas de desprecio o incomodidad. El lingüista o el educador, claro está, no pretenden usar el término de esa forma despectiva. Las villas argentinas, desde la Puna hasta la Patagonia, "tienen sucedáneos en casi todo el mundo: cantegriles, en Uruguay; barriadas, en Lima; ranchos, en Venezuela; favelas, en Brasil; callampas, en Chile; bidonvilles, en Estados Unidos; shangais, en Italia, etc." (Ratier, 1972, 10). Resulta al menos plausible la hipótesis de que los villeros hablan un dialecto social distinto del estándar. En este contexto, algunos de los chicos que hablan el dialecto estándar identifican y desprecian explícitamente el modo de hablar de los villeros.

Según Halliday (1978, 139-140), un dialecto no-estándar se caracteriza por privilegiar una orientación funcional hacia el significado que es distinta de la del estándar. El tema de que el villero es un dialecto no resulta secundario, ni siquiera en el contexto de desesperación que se vive ahora y desde hace muchos años en las villas miserias argentinas. La lingüística funcionalista nos enseña que no sólo aprendemos el lenguaje, sino que también aprendemos cosas del mundo con el lenguaje. Muchos chicos de las villas aprenden, desde muy pequeños, que hay tres clases de adolescentes y jóvenes: "chorros" (ladrones), "transas" (vendedores de droga) y "giles", es decir, "tontos" (individuos que no pertenecen a ninguno de los dos dominios anteriores). Semejante clasificación del universo humano, donde el que no roba o trafica droga es un "gil", es algo que forma parte de la orientación funcional del dialecto y algo que, con el lenguaje, se aprende desde chico.

\section{La hipótesis del estereotipo}

La desventaja para los chicos de menores recursos es todavía mayor cuando entran en juego los prejuicios sociales. Si un chico habla el dialecto no-estándar, es muy probable que los docentes lo encasillen como candidato al fracaso. Por ejemplo, el maestro y algunos compañeros creen que el chico en cuestión habla un dialecto "deficitario", por lo cual también refuerzan la idea de que su incompetencia en el estándar le impedirá avanzar con éxito en el colegio. Aunque este supuesto no suele ser "consciente" ni suele manifestarse en forma explícita, sí funciona en las interacciones educativas.

El sociólogo del lenguaje Frederick Williams $(1970,1976)$ sometió a prueba la hipótesis del estereotipo en ciertos lugares de Estados Unidos y pudo confirmar que las expectativas de los maestros con respecto al rendimiento escolar del alumno suelen corresponderse con el grado en que el dialecto de ese alumno difiere del estándar. Los estudios sociolingüísticos iniciados a mediados de la década de 1960 mantienen 
una notable vigencia. Por ejemplo, Walt Wolfram y sus colegas continúan trabajando en el análisis de los dialectos del inglés en los Estados Unidos, con particular énfasis en la educación universitaria (Wolfram et al, 1999; Kendall y Wolfram, 2016; Reaser et al., 2017).

En cada uno de los países de la América Hispana no existe por lo general una diferenciación dialectal tan marcada como en los países angloparlantes, donde el modo de hablar es un indicador mucho más fuerte del origen o de la pertenencia social. Sin embargo, también allí los adultos y los niños se representan estereotipos a partir de la variedad de lenguaje que habla un individuo: si por anticipado se cree que un chico "habla mal" y que por ello fracasará, entonces es muy probable que ese chico fracase. Ya se ha dicho no sólo los niños y jóvenes de los sectores sociales "medios" y "altos" reconocen, y generalmente desprecian, el modo de hablar de los villeros: también suelen hacerlo los docentes y los funcionarios.

En síntesis, la hipótesis del estereotipo, como la de la diferencia, contribuye a explicar por qué los chicos que no hablan el estándar tienen una mayor tendencia a fracasar en el colegio. Estos chicos no padecen una desventaja lingüística, sino una desventaja social.

\section{Conclusiones}

Sobre la base de lo expuesto hasta aquí, pueden ofrecerse las siguientes conclusiones.

\subsection{El fracaso escolar es, en principio, consecuencia de una desventaja social.}

Las hipótesis de la diferencia y del estereotipo permiten entender las serias dificultades que enfrentan los chicos de los sectores socioeconómicos menos favorecidos. De todas maneras, queda una pregunta. Si los chicos sufren a causa de su dialecto, ¿por qué no aprenden el estándar? "A los niños no les es difícil hacerlo; en muchas partes del mundo es sumamente común que un niño aprenda tres o incluso cuatro variedades de su lengua materna" (Halliday, 1978, 139). Un chico que vive en la villa y que jamás ha hecho una exposición oral en clase en lengua estándar puede imitar la variedad estándar perfectamente bien fuera de aula, por ejemplo cuando quiere hacer una broma sobre el modo de hablar de sus maestros. El problema de la dificultad de aprender el estándar tampoco radica en la escritura. El sistema de escritura castellana es neutro con respecto a las variedades regionales y sociales; se adapta tan bien al habla de una villa miseria como a la de un barrio "elegante", al castellano del Río de la Plata como al de España. No hay problemas lingüísticos especiales para aprender a leer y escribir porque se hable una variedad no estandarizada. La LSF acepta, entonces, las hipótesis de la diferencia y del estereotipo. Ambas descartan que la causa del fracaso escolar sea el sistema lingüístico que le permite hablar a un individuo. La explicación del fracaso escolar es social y, en palabras de Bernstein, "la educación no puede ser compensación de la sociedad" (Halliday, 1978, 140). 


\subsection{Sin embargo hay aspectos lingüísticos que inciden en el fracaso escolar}

La LSF acepta entonces la explicación sociológica que se sostiene en las hipótesis de la diferencia y del estereotipo, pero destaca que el problema también incluye un elemento que es esencialmente lingüístico. En el proceso educativo hay supuestos y prácticas que reflejan los valores, los patrones de comunicación y los estilos de aprendizaje de las diferentes sub-culturas. "Como ha señalado Bernstein, eso no sólo tiende a favorecer ciertos modos de aprendizaje por encima de otros, sino que también crea para algunos niños, entre el hogar y la escuela, una continuidad de cultura que en gran parte niega a otros" (Halliday, 1978, 141). El lenguaje no carece de importancia en estos procesos. ¿Cómo se transmiten si no los valores y los modos de aprendizaje privilegiados? Desde un enfoque sistémico-funcional, los "códigos" de los que habla Bernstein son diferencias de orientación dentro del potencial semiótico del lenguaje. Si el lenguaje es concebido como una "semiótica social" (un conjunto de significados disponibles a los hablantes para que produzcan mensajes significativos), entonces las diversas sub-culturas (que en total constituirían la suma de "la cultura") manejan "códigos" que conceden mayor o menor importancia a ciertos órdenes de significado. Cualquier sub-cultura particular hace hincapié en ciertas funciones o áreas del significado. Esas funciones pueden reflejar valores implícitos o explícitos. Por ejemplo, el dialecto de la villa miseria privilegia ciertas funciones y ciertos significados que no son los mismos que los del estándar, y viceversa. A través de los diferentes dialectos los hablantes van aprendiendo las orientaciones y los significados propios de su sub-cultura. En general, los significados y funciones especialmente valorados por los sectores "medios" y "altos" no son los mismos que los significados y funciones privilegiados en las villas. Tradicionalmente, los significados y las funciones relativos a la escuela fueron positivos para los hablantes del dialecto estándar. Por el contrario, sin duda por cuestiones de necesidad y urgencia, no son del todo adecuados para los villeros. Esto da una interpretación plausible del papel del lenguaje en el fracaso educativo: es un factor clave en la transmisión cultural. Si la detección del problema es complicada, su solución lo es todavía más. La escuela debería ampliar sus significados y sus orientaciones para que los chicos de los sectores socioeconómicos menos favorecidos no fueran objeto de prejuicios. Así y todo, en el corto plazo, y más allá del discurso "progresista" o "bienintencionado", lo cierto es que la escuela debería comprometerse con la enseñanza del estándar, para evitar que haya cada vez más chicos con esta desventaja lingüística.

\subsection{Debe enseñarse la variedad estándar a los chicos de sectores sociales menos favorecidos. En el prólogo al Fausto de Estanislao del Campo, Borges dice lo siguiente:}

Obras que fingen defender cosas indefendibles -Elogio de la locura, de Erasmo; Sobre el asesinato considerado como una de las bellas artes, de Thomas de Quincey; La decadencia de la mentira, de Wilde- presuponen épocas más razonables, épocas tan lejanas a la locura, al asesinato y a la mentira, que les divierte el hecho de vindicar esos males. ¿Qué pensaríamos en cambio, de épocas en las que fuera necesario probar, con dialéctica rigurosa, que el agua es superior a la sed y que la luna merece que todos los hombres la miren, siquiera una sola vez antes de morir? 
En esa época vivimos; en Buenos Aires, a mediados del siglo XX, un prólogo del Fausto debe ser, ante todo, una defensa del Fausto" (Borges, 1975, 32).

A veces da la impresión de que en efecto vivimos en una época (o en un lugar) en que debe justificarse que tomar agua es mejor que seguir con sed. Debido al importante papel que desempeña el lenguaje en el fracaso escolar, resulta imprescindible el diseño de un currículum de Lengua que se fundamente en la lingüística científica y en el que se establezcan con claridad los objetivos de los alumnos: uno de ellos tendría que ser la enseñanza o el estudio del dialecto estándar, lo cual beneficiaría en especial a los chicos provenientes de los sectores socioeconómicos menos favorecidos.

Sugerir la necesidad un currículum de base lingüística para todos los niños, "el futuro de la patria", parece algo del más elemental sentido común. Sin embargo, en estos tiempos de sed innecesaria y absurda, la propuesta de un currículum común para un sistema educativo completo puede llenar un importante vacío.

De esta forma, los exámenes finales previstos por ese currículum podrían ser comunes para todo el sistema educativo, es decir, para todos los chicos de todo el país, desde la Puna hasta la Patagonia, en el caso de Argentina. Así, los docentes sabrían qué es lo que tienen enseñar y hacia dónde dirigirse. Se garantizaría un piso común a todos los niños y adolescentes de las diferentes regiones y clases sociales. En una mirada superficial, un currículum común y evaluaciones finales bien pautadas pueden parecer esquemáticos o coercitivos, pero el objetivo buscado es contribuir a la igualdad de oportunidades.

Es un hecho bien conocido pero aún así apasionante que diferentes dialectos representan diferentes visiones de la realidad, con la consecuente capacidad de privilegiar ciertas concepciones sobre otras, por ejemplo la concepción de que la escuela es una pérdida de tiempo frente a la concepción de que la escuela es buena. En principio se trata de una cuestión cultural y no meramente lingüística. Sin embargo, la variedad de lenguaje que se habla también tiene su importancia en el asunto. Ante esta situación tan compleja, el manejo de las dos variedades (estándar y no estándar) no parece una solución por sí sola para los prejuicios o las concepciones divergentes de la escuela. En este sentido, la exposición al dialecto estándar posiblemente llevará a una situación que garantice el piso común para todos los chicos, pero esta medida será más efectiva si además se hace un esfuerzo por educar en contra del desprestigio los dialectos no estandarizados o, en otras palabras, si se hace el esfuerzo de educar en la tolerancia y a favor de la erradicación de los prejuicios.

\subsection{Se requiere una teoría funcional del lenguaje para tratar con el problema del fracaso escolar}

Permitámonos unas consideraciones últimas desde el punto de vista epistemológico. Chomsky, quien es posiblemente el lingüista más reconocido de la historia y una de las figuras intelectuales más notables de nuestra época, cree que una teoría como la LSF está mal encaminada y que por ello es globalmente falsa. Ideas propias del funcionalismo, tales como que "el lenguaje está estructurado para la comunicación" o que "está bien adaptado para sus funciones", son incompatibles con la concepción del lenguaje como facultad mental/cerebral y las condiciones para la correcta formación de oraciones. Ésta es, quizá, una de las tesis más cuestionables de Chomsky. 
Él (y la legión que lo respalda, aun con críticas de importancia) argumentan de la siguiente forma:

\section{Argumento epistemológico de Chomsky y los demás generativistas}

i. Una teoría X no explica el problema A (y otros problemas), por ejemplo, las condiciones para la correcta formación de las estructuras sintácticas a partir de la facultad del lenguaje.

ii. La teoría generativa explica A.

iii. Explicar A (y otros problemas) es un requisito para que una teoría pueda ser verdadera.

iv. Por lo tanto, la teoría $\mathrm{X}$ es falsa y la teoría generativa puede ser verdadera.

El argumento es válido. Sin embargo, la conclusión (iv) puede ser falsa porque, antes, es falsa la premisa (iii). Como dice Hempel: no hay contrastaciones o experimentos cruciales en la ciencia $(1966,51)$. Que un experimento de óptica demuestre que la luz es onda no constituye un argumento decisivo para abandonar la hipótesis de que la luz es un conjunto de corpúsculos. De manera análoga, que un análisis generativo muestre que las condiciones para la correcta formación de estructuras forman parte de la facultad mental del lenguaje no es un argumento decisivo para abandonar otras concepciones lingüísticas.

La LSF no se propone explicar las condiciones generales para la correcta formación de estructuras sintácticas sobre la base de una supuesta "facultad del lenguaje". Pero sí puede explicar otras cosas, y posiblemente por ello sea una teoría científica consolidada. A pesar de la sombra terrible de Chomsky sigue siendo necesaria una perspectiva socio-funcional para entender (y solucionar) problemas tan urgentes como el del fracaso escolar.

\section{Bibliografía}

Benveniste, Emile (1958): "Categorías de pensamiento y categorías de lengua", Problemas de Lingüistica General, I, México, Siglo XXI (1995), 63-74.

Bernstein, Basil (1971): Class, codes and control 1: Theoretical Studies towards a sociology of language, Londres, Routledge y Kegan Paul.

Borges, Jorge Luis (1975): "Estanislao del Campo, Obras Completas, IV, Buenos Aires, Emecé (1996), 31-33.

Chomsky, Noam (1995): The Minimalist Program, Cambridge y Londres, MIT Press.

Chomsky, Noam (2000): On nature and language, Nueva York y Cambridge, University Press.

Chomsky, Noam (2003): "Replies", en Chomsky and his critics, L.M. Antony y N. Hornstein (eds.), Malden, Oxford, Melbourne y Berlín, Blackwell Publishing, 255-328.

Chomsky, Noam (2005): “Three Factors in Language Design”, Linguistic Inquiry, 36 (1), $1-22$.

Córdoba Caro, Luis Gonzalo, V. García Preciado, L. Luengo Pérez, M. Vizuete Carrizosa y S. Feu Molina (2011): "Determinantes socioculturales: su relación con el rendimiento académico en alumnos de Enseñanza Secundaria Obligatoria", Revista de Investigación Educativa, 29 (1), 83-96. 
Fawcett, Robin Powell (2008): Invitation to Systemic Functional Linguistics. The Cardiff Grammar as an extension and simplification of Halliday's Systemic Functional Grammar, Beijing, Beijing University Press.

Fowler, Roger (1996): “On critical linguistics", en Texts and Practices, C. Caldas-Coulthard y M. Coulthard (comps.), Londres y Nueva York, Routledge, 1-15.

Gil, José María y A. García (2010): “Transitividad, modo y tema en español: Un primer análisis en términos de la gramática de Cardiff”, Signos, 43 (72), 71-98.

Halliday, Michael A. K. (1978): El lenguaje como semiótica social. La interpretación social del lenguaje y del significado, Bogotá, Fondo de Cultura Económica (1994).

Halliday, Michael A. K. y C. Matthiessen (2004): An Introduction to Functional Grammar, Londres, Arnold.

Hempel, Carl Gustav (1966): Filosofia de la ciencia natural, Madrid, Alianza.

Jackendoff, Ray (1997): The architecture of the language faculty, Cambridge, MIT Press.

Jackendoff, Ray (2002): Foundations of language: brain, meaning, grammar, evolution, Nueva York, Oxford University Press.

Kendall, Tyler y W. Wolfram (2016): “Engagement Through Data Management and Preservation: The North Carolina Language and Life Project and the Sociolinguistic Archive and Analysis Project", en Creating and Digitizing Language Corpora, K. Corrigan y A. Mearns (eds), Londres, Palgrave Macmillan, 133-157.

Lakatos, Imre (1974). "Falsification and the methodology of scientific research programmes", en Criticism and the growth of knowledge, I. Lakatos y A. Musgrave, A. (Comps.), Cambridge: Cambridge University Press, 91-196.

Lamb, Sydney M. (1999): Pathways of the brain: The neurocognitive basis of language, Ámsterdam, John Benjamins.

Lamb, Sydney M. (2005): "Language and Brain: When experiments are unfeasible, you have to think harder", Linguistics and the Human Sciences, 1, 151-178.

Lamb, Sydney M. (2006): "Being realistic, being scientific", LACUS Forum, 32: Networks, 201-209.

López-Martínez, Olivia y J. Navarro-Lozano. (2010): “Creatividad e inteligencia: un estudio en Educación Primaria", Revista de Investigación Educativa, 28 (2), 283-296.

Murillo Torrecilla, F. Javier y R. Hernández Castilla (2011): "Efectos escolares de factores socio-afectivos. Un estudio Multinivel para Iberoamérica", Revista de Investigación Educativa, 29 (2), 407-427.

Pinker, Stephen y R. Jackendoff (2005): “The faculty of language: what's special about it?”, Cognition, 95, 201-236.

Pinker, Stephen (1999): Words and rules: the ingredients of language, Nueva York, Harper Collins.

Ratier, Hugo (1972): Villeros y villas miseria, Buenos Aires, Centro de Editor de América Latina.

Reaser, Jeffrey, C. Adger, W. Wolfram y D. Christian (2017): Dialects at School: Educating Linguistically Diverse Students, Nueva York York: Routledge.

Santos Rego, Miguel A.; M. Lorenzo Moledo y D. Priegue Caamaño (2011): "Infancia de la inmigración y educación: la visión de las familias", Revista de Investigación Educativa, 29 (1), 97-110.

Sapir, Edward (1921): El lenguaje, México, Fondo de Cultura Económica (1994).

Trevitt, Chris, E. Breman y C. Stocks (2012): "Assessment and learning: is it time to rethink student activities and academic roles?", Revista de Investigación Educativa, 30 (2), 253267. 
Williams, Frederic (1970): Language and poverty: perspectives on a theme, Chicago, Markham.

Williams, Frederic (1976): Explorations of the linguistic attitudes of teachers, Rowley, Newbury House Publishers.

Wolfram, Walt, C. T. Adger y D. Christian (1999): Dialects in schools and communities, Mahwah, Lawrence Erlbaum Associates. 